\title{
An Approach to Multiple Attribute Group Decision Making Based on Intuitionistic Trapezoidal Fuzzy Power Generalized Aggregation Operator
}

\author{
Peide Liu ${ }^{*}$, Ying Liu \\ School of Management Science and Engineering, Shandong University of Finance and Economics No. 7366, Erhuandong \\ Road \\ Jinan, Shandong 250014, China
}

Received 2 May 2012

Accepted 11 November 2012

\begin{abstract}
With respect to multiple attribute decision making (MADM) problems in which the attribute value takes the form of intuitionistic trapezoidal fuzzy number, a new decision making analysis method is developed. Firstly, some operational laws and expected values of intuitionistic trapezoidal fuzzy numbers, and distance between two intuitionistic trapezoidal fuzzy numbers, are introduced, and the comparison method for the intuitionistic trapezoidal fuzzy numbers is proposed. Then, combined the power aggregation operator and the generalized aggregation operator, a power generalized average (PGA) operator is proposed, and some properties of the PGA operator, such as idempotency, boundary, commutativity, etc., are studied. At the same time, some special cases of the generalized parameters in PGA operator are analyzed. Furthermore, an intuitionistic trapezoidal fuzzy power generalized weighted average (ITFPGWA) operator is also proposed for the intuitionistic trapezoidal fuzzy information, and some properties of the ITFPGWA operator and an approach to deal with group decision making problems under intuitionistic trapezoidal fuzzy information based on the ITFPGWA operator are given. Finally, an illustrative example is given to illustrate the decision-making steps, and to demonstrate its practicality and effectiveness.
\end{abstract}

Keywords: Decision analysis; multiple criteria analysis; the intuitionistic trapezoidal fuzzy number; multiple attribute group decision making; the power generalized aggregation operator.

\section{Introduction}

Multiple attribute decision making (MADM) problems are the important research contents of decision theory. Since the concept of fuzzy sets was proposed by Zadeh ${ }^{1}$, the significant achievements about the theory research and applications of fuzzy sets have been made. However, the fuzzy set is used to characterize the fuzziness just by membership degree. Atanassov ${ }^{2,3}$ proposed the intuitionistic fuzzy set (IFS) characterized by a membership function and a non-membership function, which is a generalization of the concept of fuzzy set. $\mathrm{Xu}$ and Yager $^{4}, \mathrm{Xu}^{5}$ proposed some aggregation operators with intuitionistic fuzzy information. Later, Atanassov and Gargov ${ }^{6}$, Atanassov ${ }^{7}$ further introduced the interval-valued intuitionistic fuzzy set (IVIFS), which is a generalization of the IFS. The fundamental characteristic of the IVIFS is that the values of its membership function and non-membership function are interval numbers rather than crisp numbers. $\mathrm{Xu}^{8}, \mathrm{Xu}$ and $\mathrm{Chen}^{9}$ further proposed some aggregation operators with interval-valued intuitionistic fuzzy

\footnotetext{
* Corresponding author: Peide Liu. Email: Peide.liu@gmail.com, Tel:+86-531-88525938. The present address: No.7366, Erhuandong Road, Jinan 250014, Shandong Province, China. The affiliation: School of Management Science and Engineering, Shandong University of Finance and Economics
} 
information. Shu et al. ${ }^{10}$ gave the definition and operational laws of intuitionistic triangular fuzzy number and proposed a decision method with intuitionistic triangular fuzzy information. Zhang and $\mathrm{Liu}^{11}$ proposed the triangular intuitionistic fuzzy number which used the triangular fuzzy number to denote the membership degree and the non-membership degree, then the weighted arithmetic average operator was defined, and an approach to multiple attribute group decision making (MAGDM) with triangular intuitionistic fuzzy information was developed. Furthermore, Wang ${ }^{12}$, Wang and Zhang ${ }^{13}$ gave the definition of intuitionistic trapezoidal fuzzy number and interval intuitionistic trapezoidal fuzzy number, and defined the expected values of intuitionistic trapezoidal fuzzy number and proposed a decision method based on intuitionistic trapezoidal fuzzy number. Wang and Zhang $^{14}$ proposed the Hamming distance of intuitionistic trapezoidal fuzzy numbers and intuitionistic trapezoidal fuzzy weighted arithmetic averaging (ITFWAA) operator, then proposed multicriteria decision-making method with incomplete certain information. Wang and Zhang ${ }^{15}$ proposed some aggregation operators, including intuitionistic trapezoidal fuzzy weighted arithmetic averaging operator and weighted geometric averaging operator, and expected values, score function and accuracy function of intuitionitsic trapezoidal fuzzy numbers were defined, and a ranking of the alternatives can be attained. Wan and Dong ${ }^{16}$ proposed the expectation and expectant score by the coordinates of gravity center of intuitionistic trapezoidal fuzzy number, and defined ordered weighted aggregation operator and hybrid aggregation operator for intuitionistic trapezoidal fuzzy numbers. Wei ${ }^{17}$ proposed some aggregation operators, including intuitionistic trapezoidal fuzzy ordered weighted averaging (ITFOWA) operator and intuitionistic trapezoidal fuzzy hybrid aggregation (ITFHA) operator, and developed an approach to multiple attribute group decision making (MAGDM) with intuitionistic trapezoidal fuzzy information.

The information aggregation operators are an interesting research topic, which is receiving increasing concern. Yager $^{18}$ proposed the generalized ordered weighted averaging (GOWA) operator which is an extension of the OWA operator. $\mathrm{Li}^{19}$, Zhao et al. ${ }^{20}$ further proposed the generalized aggregation operators for intuitionistic fuzzy sets. Merigó and Casanovas ${ }^{21}$ presented the generalized hybrid averaging (GHA) operator. It is able to generalize a wide range of mean operators such as the HA, the hybrid geometric averaging (HGA), the hybrid quadratic averaging (HQA), the generalized ordered weighted averaging (GOWA) operator and the weighted generalized mean (WGM). A key feature in GHA operator is that it is able to deal with the weighted average and the ordered weighted averaging (OWA) operator in the same formulation. Merigó and Casanovas ${ }^{22}$ introduced the fuzzy generalized hybrid averaging (FGHA) operator for the multi-attribute decision-making problems in which the attribute values take the form of the fuzzy number; this expanded the application scope of GHA operator. However, most of the existing aggregation operators do not take into account the relationship between the values being fused. Yager $^{23}$ developed a power-average (PA) operator and a power OWA (POWA) operator, whose weighting vectors depend upon the input arguments and allow values being aggregated to support and reinforce each other. $\mathrm{Xu}$ and Yager $^{24}$ developed a power-geometric (PG) operator, a power-ordered-geometric (POG) operator and a powerordered-weighted-geometric (POWG) operator, and studied some properties of these operators. Then, an uncertain PG (UPG) operator and its weighted form, and an uncertain power-ordered-weighted-geometric (UPOWG) operator are proposed to aggregate the input arguments taking the form of interval of numerical values, and the approaches to group decision making are developed based on these operators. $\mathrm{Xu}^{25}$ developed a series of operators for aggregating the intuitionistic fuzzy numbers, and established various properties of these power aggregation operators, and then some approaches to multiple attribute group decision making with intuitionistic fuzzy information and interval-valued intuitionistic fuzzy information were proposed. $\mathrm{Xu}$ and Wang $^{26}$ developed some new linguistic aggregation operators, such as 2-tuple linguistic power average (2TLPA) operator, 2-tuple linguistic weighted PA operator, 2TLPOWA operator which are based on PA operator, then studied some properties of these operators, such as idempotency, boundary, etc. Moreover, two approaches to deal with group decision making problems under linguistic environment were developed. 
The intuitionistic trapezoidal fuzzy numbers are very suitable to be used for depicting uncertain or fuzzy information. Motivated by the idea of power aggregation operator proposed by Yager $^{23}$ and the generalized aggregation operators proposed by Yager $^{18}$ and Zhao et $\mathrm{al}^{20}$, this paper is to propose some operators, such as a power generalized average (PGA) operator and an intuitionistic trapezoidal fuzzy power generalized weighted average (ITFPGWA) operator, and study some properties of these operators, such as idempotency, boundary, commutativity, etc. Then propose an approach to deal with group decision making problems under intuitionistic trapezoidal fuzzy information.

\section{Preliminaries}

\subsection{The intuitionistic trapezoidal fuzzy numbers}

In the following, we shall introduce some basic concepts related to intuitionistic trapezoidal fuzzy numbers.

Definition $1^{12-15}$ : Let $\tilde{a}$ be an intuitionistic trapezoidal fuzzy numbers (ITFNs), its membership function is defined as

$$
\mu_{\tilde{a}}(x)= \begin{cases}\frac{x-a}{b-a} \mu_{\tilde{a}}, & a \leq x<b \\ \mu_{\tilde{a}}, & b \leq x \leq c \\ \frac{d-x}{d-c} \mu_{\tilde{a}}, & c<x \leq d \\ 0, & \text { otherwise }\end{cases}
$$

Its non-membership function is defined as

$$
v_{\tilde{a}}(x)= \begin{cases}\frac{(b-x)+v_{\tilde{a}}\left(x-a_{1}\right)}{b-a_{1}}, & a_{1} \leq x<b \\ v_{\tilde{a}}, & b \leq x \leq c \\ \frac{(x-c)+v_{\tilde{a}}\left(d_{1}-x\right)}{d_{1}-c}, & c<x \leq d_{1} \\ 0, & \text { otherwise }\end{cases}
$$

where, $\quad 0 \leq \mu_{\tilde{a}} \leq 1 \quad, \quad 0 \leq v_{\tilde{a}} \leq 1 \quad$ and $0 \leq \mu_{\tilde{a}}+v_{\tilde{a}} \leq 1 \quad ; \quad a, a_{1}, b, c, d, d_{1} \in R \quad$. The intuitionistic fuzzy number is denoted as $\tilde{a}=\left\langle\left([a, b, c, d] ; \mu_{\tilde{a}}\right),\left(\left[a_{1}, b, c, d_{1}\right] ; v_{\tilde{a}}\right)\right\rangle$.Different from fuzzy numbers, the intuitionistic fuzzy numbers have another parameter: non-membership function, which is used to express the extent to which the decision makers think that the element does not belong to $\left[a_{1}, b, c, d_{1}\right]$. When $\mu_{\tilde{a}}=1, v_{\tilde{a}}=0, \tilde{a}$ is changed into trapezoidal fuzzy number. Generally, there is $[a, b, c, d]=\left[a_{1}, b, c, d_{1}\right]$ in intuitionistic trapezoidal fuzzy number $\tilde{a}$, here, denoted as $\tilde{a}=\left([a, b, c, d] ; \mu_{\tilde{a}}, v_{\tilde{a}}\right)$.

Definition $2^{12-15}$. Let $\tilde{a}_{1}=\left(\left[a_{1}, b_{1}, c_{1}, d_{1}\right] ; \mu_{\tilde{a}_{1}}, v_{\tilde{a}_{1}}\right)$ and $\tilde{a}_{2}=\left(\left[a_{2}, b_{2}, c_{2}, d_{2}\right] ; \mu_{\tilde{a}_{2}}, v_{\tilde{a}_{2}}\right)$ be two intuitionistic trapezoidal fuzzy numbers, and $\lambda \geq 0$, then

(1)

$$
\begin{aligned}
& \tilde{a}_{1}+\tilde{a}_{2}=\left(\left[a_{1}+a_{2}, b_{1}+b_{2}, c_{1}+c_{2}, d_{1}+d_{2}\right] ;\right. \\
& \left.\mu_{\tilde{a}_{1}}+\mu_{\tilde{a}_{2}}-\mu_{\tilde{a}_{1}} \mu_{\tilde{a}_{2}}, v_{\tilde{a}_{1}} v_{\tilde{a}_{2}}\right) \\
& \tilde{a}_{1} \otimes \tilde{a}_{2}=\left(\left[a_{1} a_{2}, b_{1} b_{2}, c_{1} c_{2}, d_{1} d_{2}\right] ;\right. \\
& \left.\mu_{\tilde{a}_{1}} \mu_{\tilde{a}_{2}}, v_{\tilde{a}_{1}}+v_{\tilde{a}_{2}}-v_{\tilde{a}_{1}} v_{\tilde{a}_{2}}\right) \\
& \text { (3) } \lambda \tilde{a}_{1}=\left(\left[\lambda a_{1}, \lambda b_{1}, \lambda c_{1}, \lambda d_{1}\right] ; 1-\left(1-\mu_{\tilde{a}_{1}}\right)^{\lambda}, v_{\tilde{a}_{1}}^{\lambda}\right) \\
& \text { (4) } \tilde{a}_{1}^{\lambda}=\left(\left[a_{1}^{\lambda}, b_{1}^{\lambda} c_{1}^{\lambda} d_{1}^{\lambda}\right] ; \mu_{\tilde{a}_{1}}^{\lambda}, 1-\left(1-v_{\tilde{a}_{1}}\right)^{\lambda}\right)
\end{aligned}
$$$$
\text { (2) }
$$

Theorem $1^{12-15}$ : Let $\tilde{a}_{1}=\left(\left[a_{1}, b_{1}, c_{1}, d_{1}\right] ; \mu_{\tilde{a}_{1}}, v_{\tilde{a}_{1}}\right)$ and $\tilde{a}_{2}=\left(\left[a_{2}, b_{2}, c_{2}, d_{2}\right] ; \mu_{\tilde{a}_{2}}, v_{\tilde{a}_{2}}\right)$ be two intuitionistic trapezoidal fuzzy numbers, then the calculation rules between $\tilde{a}_{1}$ and $\tilde{a}_{2}$ are shown as follows:

(1) $\tilde{a}_{1}+\tilde{a}_{2}=\tilde{a}_{2}+\tilde{a}_{1}$

(2) $\tilde{a}_{1} \otimes \tilde{a}_{2}=\tilde{a}_{2} \otimes \tilde{a}_{1}$

(3) $\lambda\left(\tilde{a}_{1}+\tilde{a}_{2}\right)=\lambda \tilde{a}_{1}+\lambda \tilde{a}_{2}, \lambda \geq 0$

(4) $\lambda_{1} \tilde{a}_{1}+\lambda_{2} \tilde{a}_{1}=\left(\lambda_{1}+\lambda_{2}\right) \tilde{a}_{1}, \lambda_{1}, \lambda_{2} \geq 0$

(5) $\tilde{a}_{1}^{\lambda_{1}} \otimes \tilde{a}_{1}^{\lambda_{2}}=\left(\tilde{a}_{1}\right)^{\lambda_{1}+\lambda_{2}}, \lambda_{1}, \lambda_{2} \geq 0$

(6) $\tilde{a}_{1}^{\lambda_{1}} \otimes \tilde{a}_{2}^{\lambda_{1}}=\left(\tilde{a}_{1} \otimes \tilde{a}_{2}\right)^{\lambda_{1}}, \lambda_{1} \geq 0$

Theorem $2^{15}$ : For the intuitionistic trapezoidal fuzzy number $\tilde{a}=\left([a, b, c, d] ; \mu_{\tilde{a}}, v_{\tilde{a}}\right)$, its expected value is shown as follows:

$$
I(\tilde{a})=\frac{1}{8} \times\left[(a+b+c+d) \times\left(1+\mu_{\tilde{a}}-v_{\tilde{a}}\right)\right]
$$


Definition $3^{14}$ : Let $\tilde{a}_{1}=\left(\left[a_{1}, b_{1}, c_{1}, d_{1}\right] ; \mu_{\tilde{a}_{1}}, v_{\tilde{a}_{1}}\right)$ and $\tilde{a}_{2}=\left(\left[a_{2}, b_{2}, c_{2}, d_{2}\right] ; \mu_{\tilde{a}_{2}}, v_{\tilde{a}_{2}}\right)$ be two intuitionistic trapezoidal fuzzy numbers, then the normalized Hamming distance between $\tilde{a}_{1}$ and $\tilde{a}_{2}$ is defined as follows:

$$
\begin{aligned}
d\left(\tilde{a}_{1}, \tilde{a}_{2}\right)= & \frac{1}{8}\left(\left|\left(1+\mu_{\tilde{a}_{1}}-v_{\tilde{a}_{1}}\right) a_{1}-\left(1+\mu_{\tilde{a}_{2}}-v_{\tilde{a}_{2}}\right) a_{2}\right|\right. \\
& +\left|\left(1+\mu_{\tilde{a}_{1}}-v_{\tilde{a}_{1}}\right) b_{1}-\left(1+\mu_{\tilde{a}_{2}}-v_{\tilde{a}_{2}}\right) b_{2}\right| \\
& +\left|\left(1+\mu_{\tilde{a}_{1}}-v_{\tilde{a}_{1}}\right) c_{1}-\left(1+\mu_{\tilde{a}_{2}}-v_{\tilde{a}_{2}}\right) c_{2}\right| \\
& \left.+\left|\left(1+\mu_{\tilde{a}_{1}}-v_{\tilde{a}_{1}}\right) d_{1}-\left(1+\mu_{\tilde{a}_{2}}-v_{\tilde{a}_{2}}\right) d_{2}\right|\right)
\end{aligned}
$$

Definition $4^{15}$. Let $\tilde{a}=\left([a, b, c, d] ; \mu_{\tilde{a}}, v_{\tilde{a}}\right)$ be an intuitionistic trapezoidal fuzzy number; then, $S(\tilde{a})=I(\tilde{a}) \times\left(\mu_{\tilde{a}}-v_{\tilde{a}}\right)$ is called the score function of $\tilde{a}$, where $I(\tilde{a})$ is the expected value in definition 3 .

Definition $5^{15}$. Let $\tilde{a}=\left([a, b, c, d] ; \mu_{\tilde{a}}, v_{\tilde{a}}\right)$ be an intuitionistic trapezoidal fuzzy number; then, $H(\tilde{a})=I(\tilde{a}) \times\left(\mu_{\tilde{a}}+v_{\tilde{a}}\right)$ is called the accuracy function, where $I(\tilde{a})$ is the expected value in definition 3.

Definition 6. If $\tilde{a}_{1}$ and $\tilde{a}_{2}$ are any two intuitionistic trapezoidal fuzzy numbers, then,

(1) If $I\left(\tilde{a}_{1}\right)>I\left(\tilde{a}_{2}\right)$, then $\tilde{a}_{1} \succ \tilde{a}_{2}$;

(2) If $I\left(\tilde{a}_{1}\right)=I\left(\tilde{a}_{2}\right)$, and

$$
\text { If } S\left(\tilde{a}_{1}\right)>S\left(\tilde{a}_{2}\right) \text { then } \tilde{a}_{1} \succ \tilde{a}_{2} \text {; }
$$$$
\text { If } S\left(\tilde{a}_{1}\right)=S\left(\tilde{a}_{2}\right) \text { and }
$$

$$
\begin{aligned}
& \text { If } H\left(\tilde{a}_{1}\right)>H\left(\tilde{a}_{2}\right) \text { then } \tilde{a}_{1} \succ \tilde{a}_{2} ; \\
& \text { If } H\left(\tilde{a}_{1}\right)=H\left(\tilde{a}_{2}\right) \text { then } \tilde{a}_{1}=\tilde{a}_{2} .
\end{aligned}
$$

\subsection{The power operator}

Yager $^{23}$ introduced a nonlinear weighted-average aggregation tool, which is called power average (PA) operator, and which can be defined as follows:

$$
P A\left(a_{1}, a_{2}, \cdots, a_{n}\right)=\frac{\sum_{i=1}^{n}\left(1+T\left(a_{i}\right)\right) a_{i}}{\sum_{i=1}^{n}\left(1+T\left(a_{i}\right)\right)}
$$

$$
T\left(a_{i}\right)=\sum_{\substack{j=1 \\ j \neq i}}^{n} \operatorname{Sup}\left(a_{i}, a_{j}\right)
$$

and $\operatorname{Sup}(a, b)$ is considered as the support for $a$ from $b$, which satisfies the following three properties.

1) $\operatorname{Sup}(a, b) \in[0,1]$.

2) $\operatorname{Sup}(a, b)=\operatorname{Sup}(b, a)$.

3) $\operatorname{Sup}(a, b) \geq \operatorname{Sup}(x, y)$, if ||$a-b|<| x-y \mid$.

So, we can get the more the similarity, the closer the two values are, and the more they support each other.

$$
\text { Let } V_{i}=1+T\left(a_{i}\right) \text {, and } w_{i}=V_{i} / \sum_{i=1}^{n} V_{i} \text {, then } w_{i}
$$

meets $w_{i} \in[0,1], \sum_{i=1}^{n} w_{i}=1$. So, PA operator can be expressed as follows

$$
P A\left(a_{1}, a_{2}, \cdots, a_{n}\right)=\sum_{i=1}^{n} w_{i} a_{i}
$$

The PGA operator is a generalization of the PA operator by using generalized means. It can be defined as follows.

Definition 7. A PGA operator of dimension $n$ is a mapping PGA: $R^{n} \rightarrow R$. Such that,

$$
\operatorname{PGA}\left(a_{1}, a_{2}, \cdots, a_{n}\right)=\left(\sum_{j=1}^{n} w_{j} a_{j}^{\lambda}\right)^{1 / \lambda}
$$

where $\quad w_{i}=V_{i} / \sum_{i=1}^{n} V_{i} \quad, \quad V_{i}=1+T\left(a_{i}\right) \quad$ and $T\left(a_{i}\right)=\sum_{\substack{j=1 \\ j \neq i}}^{n} \operatorname{Sup}\left(a_{i}, a_{j}\right)$. In addition, $\lambda$ is a parameter such that $\lambda \in(-\infty, 0) \cup(0,+\infty)$.

Some properties of the PGA operators are shown as follows.

(1) When $\lambda \rightarrow-\infty$, then

$\operatorname{PGA}\left(a_{1}, a_{2}, \cdots, a_{n}\right)=\min \left(a_{1}, a_{2}, \cdots, a_{n}\right)$.

(2) When $\lambda \rightarrow 0, \operatorname{PGA}\left(a_{1}, a_{2}, \cdots, a_{n}\right)=\prod_{j=1}^{n} a_{j}^{w_{j}}$.

The PGA operator reduces to the PG operator.

where 
(3) When $\lambda=1, \operatorname{PGA}\left(a_{1}, a_{2}, \cdots, a_{n}\right)=\sum_{j=1}^{n} w_{j} a_{j}$.

The PGA operator reduces to the PA operator.

(4) When $\lambda \rightarrow+\infty$, then

$\operatorname{PGA}\left(a_{1}, a_{2}, \cdots, a_{n}\right)=\max \left(a_{1}, a_{2}, \cdots, a_{n}\right)$.

The $P G A$ operator has the following properties:

(1) Theorem 3 (Commutativity).

Let $\left(a_{1}^{\prime}, a_{2}^{\prime}, \cdots, a_{n}^{\prime}\right)$ be any permutation of $\left(a_{1}, a_{2}, \cdots, a_{n}\right)$, then

$$
P G A\left(a_{1}^{\prime}, a_{2}^{\prime}, \cdots, a_{n}^{\prime}\right)=P G A\left(a_{1}, a_{2}, \cdots, a_{n}\right)
$$

Proof. Let

$$
\begin{aligned}
& \operatorname{PGA}\left(a_{1}, a_{2}, \cdots, a_{n}\right)=\left(\sum_{j=1}^{n} w_{j} a_{j}^{\lambda}\right)^{1 / \lambda}=\left(\frac{\sum_{j=1}^{n}\left(1+T\left(a_{j}\right)\right) a_{j}^{\lambda}}{\sum_{j=1}^{n}\left(1+T\left(a_{j}\right)\right)}\right)^{1 / \lambda} \\
& \operatorname{PGA}\left(a_{1}^{\prime}, a_{2}^{\prime}, \cdots, a_{n}^{\prime}\right)=\left(\sum_{j=1}^{n} w_{j} a_{j}^{\prime \lambda}\right)^{1 / \lambda}=\left(\frac{\sum_{j=1}^{n}\left(1+T\left(a_{j}^{\prime}\right)\right) a_{j}^{\prime \lambda}}{\sum_{j=1}^{n}\left(1+T\left(a_{j}^{\prime}\right)\right)}\right)^{1 / \lambda}
\end{aligned}
$$

Since $\left(a_{1}^{\prime}, a_{2}^{\prime}, \cdots, a_{n}^{\prime}\right) \quad$ is any permutation of $\left(a_{1}, a_{2}, \cdots, a_{n}\right)$, we have

$$
\begin{aligned}
& \sum_{j=1}^{n}\left(1+T\left(a_{j}\right)\right)=\sum_{j=1}^{n}\left(1+T\left(a_{j}^{\prime}\right)\right), \\
& \sum_{j=1}^{n}\left(1+T\left(a_{j}\right)\right) a_{j}^{\lambda}=\sum_{j=1}^{n}\left(1+T\left(a_{j}^{\prime}\right)\right) a_{j}^{\prime \lambda}
\end{aligned}
$$

thus

$$
P G A\left(a_{1}^{\prime}, a_{2}^{\prime}, \cdots, a_{n}^{\prime}\right)=P G A\left(a_{1}, a_{2}, \cdots, a_{n}\right) .
$$

\section{(2) Theorem4 (Idempotency)}

Let $a_{j}=a, j=1,2, \cdots, n$, then $\operatorname{PGA}\left(a_{1}, a_{2}, \cdots, a_{n}\right)=a$

Proof. Since $a_{j}=a$, for all $j$, we have

$$
\begin{aligned}
& \operatorname{PGA}\left(a_{1}, a_{2}, \cdots, a_{n}\right)=\left(\sum_{j=1}^{n} w_{j} a_{j}^{\lambda}\right)^{1 / \lambda}=\left(\frac{\sum_{j=1}^{n}\left(1+T\left(a_{j}\right)\right) a_{j}^{\lambda}}{\sum_{j=1}^{n}\left(1+T\left(a_{j}\right)\right)}\right)^{1 / \lambda} \\
& =\left(\frac{\sum_{j=1}^{n}\left(1+T\left(a_{j}\right)\right) a^{\lambda}}{\sum_{j=1}^{n}\left(1+T\left(a_{j}\right)\right)}\right)^{1 / \lambda}=\left(\frac{a^{\lambda} \sum_{j=1}^{n}\left(1+T\left(a_{j}\right)\right)}{\sum_{j=1}^{n}\left(1+T\left(a_{j}\right)\right)}\right)^{1 / \lambda}
\end{aligned}
$$

$=\left(a^{\lambda}\right)^{1 / \lambda}=a$

\section{(3) Theorem 5 (Boundedness)}

The $P G A$ operator lies between the max and min operators:

$\min \left(a_{1}, a_{2}, \cdots, a_{n}\right) \leq P G A\left(a_{1}, a_{2}, \cdots, a_{n}\right) \leq \max \left(a_{1}, a_{2}, \cdots, a_{n}\right)$

\section{Proof.}

Let $a=\min \left(a_{1}, a_{2}, \cdots, a_{n}\right), b=\max \left(a_{1}, a_{2}, \cdots, a_{n}\right)$.

Since $a \leq a_{j} \leq b$, then

$$
\begin{aligned}
\left(\frac{\sum_{j=1}^{n}\left(1+T\left(a_{j}\right)\right) a^{\lambda}}{\sum_{j=1}^{n}\left(1+T\left(a_{j}\right)\right)}\right)^{1 / \lambda} & \leq\left(\frac{\sum_{j=1}^{n}\left(1+T\left(a_{j}\right)\right) a_{j}^{\lambda}}{\sum_{j=1}^{n}\left(1+T\left(a_{j}\right)\right)}\right)^{1 / \lambda} \\
& \leq\left(\frac{\sum_{j=1}^{n}\left(1+T\left(a_{j}\right)\right) b^{\lambda}}{\sum_{j=1}^{n}\left(1+T\left(a_{j}\right)\right)}\right)^{1 / \lambda}
\end{aligned}
$$

That is

$$
a \leq\left(\frac{\sum_{j=1}^{n}\left(1+T\left(a_{j}\right)\right) a_{j}^{\lambda}}{\sum_{j=1}^{n}\left(1+T\left(a_{j}\right)\right)}\right)^{1 / \lambda} \leq b
$$

i.e. $\min \left(a_{1}, a_{2}, \cdots, a_{n}\right) \leq P G A\left(a_{1}, a_{2}, \cdots, a_{n}\right) \leq \max \left(a_{1}, a_{2}, \cdots, a_{n}\right)$

In the preceding, we assumed that all of the objects $\left(a_{1}, a_{2}, \cdots, a_{n}\right)$ being aggregated were of equal importance. Here, we shall consider the effect on the power operations of having differing importance of the objects. Assume that each being aggregated has a weight $\omega_{i} \in[0,1]$ indicating its importance. The procedure for including this importance involves a simple modification of the value $V_{i}$ for definition 7 . It is defined as

$$
V_{i}=\omega_{i}\left(1+T\left(a_{i}\right)\right), T\left(a_{i}\right)=\sum_{\substack{j=1 \\ j \neq i}}^{n} \operatorname{Sup}\left(a_{i}, a_{j}\right)
$$

\section{Intuitionistic Trapezoidal Fuzzy Power Generalized Aggregation Operator}

Let $\tilde{a}_{j}=\left(\left[a_{j}{ }^{1}, a_{j}{ }^{2}, a_{j}{ }^{3}, a_{j}{ }^{4}\right] ; \mu_{j}, v_{j}\right)$ be a collection of ITFNs, and $\omega=\left(\omega_{1}, \omega_{2}, \cdots, \omega_{n}\right)^{T}$ be the weight 
vector of $\quad \tilde{a}_{j}(j=1,2, \cdots, n) \quad, \quad$ where $\omega_{j} \geq 0, j=1,2, \cdots, n$ and $\sum_{j=1}^{n} \omega_{j}=1$, then we define an intuitionistic trapezoidal fuzzy power generalized weighted average (ITFPGWA) operator as follows:

$\operatorname{ITFPGWA}\left(\tilde{a}_{1}, \tilde{a}_{2}, \cdots, \tilde{a}_{n}\right)=\left(\frac{\sum_{j=1}^{n} \omega_{j}\left(1+T\left(\tilde{a}_{j}\right)\right) \tilde{a}_{j}^{\lambda}}{\sum_{j=1}^{n} \omega_{j}\left(1+T\left(\tilde{a}_{j}\right)\right)}\right)^{1 / \lambda}$

Where $\lambda \in(0,+\infty)$.

According to Definition 2, formula (20) can be transformed into the following form by using mathematical induction on $n$ :

$\operatorname{ITFPGWA}\left(\tilde{a}_{1}, \tilde{a}_{2}, \cdots, \tilde{a}_{n}\right)$

$$
\begin{gathered}
=\left(\mid \frac{\sum_{j=1}^{n} \omega_{j}\left(1+T\left(\tilde{a}_{j}\right)\right) a_{j}^{1 \lambda}}{\sum_{j=1}^{n} \omega_{j}\left(1+T\left(\tilde{a}_{j}\right)\right)}\right)^{1 / \lambda},\left(\frac{\sum_{j=1}^{n} \omega_{j}\left(1+T\left(\tilde{a}_{j}\right)\right) a_{j}^{2 \lambda}}{\sum_{j=1}^{n} \omega_{j}\left(1+T\left(\tilde{a}_{j}\right)\right)}\right)^{1 / \lambda}, \\
\left.\left(\frac{\sum_{j=1}^{n} \omega_{j}\left(1+T\left(\tilde{a}_{j}\right)\right) a_{j}^{3 \lambda}}{\sum_{j=1}^{n} \omega_{j}\left(1+T\left(\tilde{a}_{j}\right)\right)}\right)^{1 / \lambda},\left(\frac{\sum_{j=1}^{n} \omega_{j}\left(1+T\left(\tilde{a}_{j}\right)\right) a_{j}^{4 \lambda}}{\sum_{j=1}^{n} \omega_{j}\left(1+T\left(\tilde{a}_{j}\right)\right)}\right)^{1 / \lambda}\right] \\
\\
\left(1-\left(\prod_{j=1}^{n}\left(1-u_{j}^{\lambda}\right)_{j=1}^{\frac{\omega_{j}\left(1+T\left(\tilde{a}_{j}\right)\right)}{n} \omega_{j}\left(1+T\left(\tilde{a}_{j}\right)\right)}\right)\right)^{1 / \lambda}, \\
\left.1-\left(1-\prod_{j=1}^{n}\left(1-\left(1-v_{j}\right)^{\lambda}\right)_{j=1}^{\frac{\omega_{j}\left(1+T\left(\tilde{a}_{j}\right)\right)}{\sum_{j}^{n} \omega_{j}\left(1+T\left(\tilde{a}_{j}\right)\right)}}\right)^{1 / \lambda}\right)
\end{gathered}
$$

Where $T\left(\tilde{a}_{i}\right)=\sum_{\substack{j=1 \\ j \neq i}}^{n} \operatorname{Sup}\left(\tilde{a}_{i}, \tilde{a}_{j}\right)$ and $\operatorname{Sup}\left(\tilde{a}_{i}, \tilde{a}_{j}\right)$ is considered as the support for $\tilde{a}_{i}$ from $\tilde{a}_{j}$, which satisfies the following three properties.

1) $\operatorname{Sup}\left(\tilde{a}_{i}, \tilde{a}_{j}\right) \in[0,1]$.

2) $\operatorname{Sup}\left(\tilde{a}_{i}, \tilde{a}_{j}\right)=\operatorname{Sup}\left(\tilde{a}_{j}, \tilde{a}_{i}\right)$.
3) $\operatorname{Sup}\left(\tilde{a}_{i}, \tilde{a}_{j}\right) \geq \operatorname{Sup}\left(\tilde{a}_{k}, \tilde{a}_{t}\right)$, if $d\left(\tilde{a}_{i}, \tilde{a}_{j}\right)<d\left(\tilde{a}_{k}, \tilde{a}_{t}\right)$.

where $d$ is a distance measure in definition 3 .

Some special cases of the ITFGWA operator are shown as follows.

(1) When $\lambda \rightarrow 0$,

$\operatorname{ITFPGWA}\left(\tilde{a}_{1}, \tilde{a}_{2}, \cdots, \tilde{a}_{n}\right)$

$=\left(\left[\prod_{j=1}^{n} a_{j}^{1} \frac{\omega_{j}\left(1+T\left(\tilde{a}_{j}\right)\right)}{\sum_{j=1}^{n} \omega_{j}\left(1+T\left(\tilde{a}_{j}\right)\right)}, \prod_{j=1}^{n} a_{j}^{2} \frac{\omega_{j}\left(1+T\left(\tilde{a}_{j}\right)\right)}{\sum_{j=1}^{n} \omega_{j}\left(1+T\left(\tilde{a}_{j}\right)\right)}\right.\right.$,

$\left.\prod_{j=1}^{n} a_{j}^{\frac{\omega_{j}\left(1+T\left(\tilde{a}_{j}\right)\right)}{\sum_{j=1}^{n} \omega_{j}\left(1+T\left(\tilde{a}_{j}\right)\right)}}, \prod_{j=1}^{n} a_{j}^{4} \frac{\omega_{j=1}\left(1+T\left(\tilde{a}_{j}\right)\right)}{\sum_{j=1}^{n} \omega_{j}\left(1+T\left(\tilde{a}_{j}\right)\right)}\right]$

$$
\left.\prod_{j=1}^{n} u_{j}^{\frac{\omega_{j}\left(1+T\left(\tilde{a}_{j}\right)\right)}{\sum_{j=1}^{n} \omega_{j}\left(1+T\left(\tilde{a}_{j}\right)\right)}}, 1-\prod_{j=1}^{n}\left(1-v_{j}\right) \frac{\omega_{j}\left(1+T\left(\tilde{a}_{j}\right)\right)}{\sum_{j=1}^{n} \omega_{j}\left(1+T\left(\tilde{a}_{j}\right)\right)}\right)
$$

then the ITFPGWA operator reduces to the ITFPWG operator.

(2) When $\lambda=1$,

$\operatorname{ITFPGWA}\left(\tilde{a}_{1}, \tilde{a}_{2}, \cdots, \tilde{a}_{n}\right)$

$$
\begin{aligned}
= & \left(\left[\frac{\sum_{j=1}^{n} \omega_{j}\left(1+T\left(\tilde{a}_{j}\right)\right) a_{j}^{1}}{\sum_{j=1}^{n} \omega_{j}\left(1+T\left(\tilde{a}_{j}\right)\right)}, \frac{\sum_{j=1}^{n} \omega_{j}\left(1+T\left(\tilde{a}_{j}\right)\right) a_{j}^{2}}{\sum_{j=1}^{n} \omega_{j}\left(1+T\left(\tilde{a}_{j}\right)\right)},\right.\right. \\
& \left.\frac{\sum_{j=1}^{n} \omega_{j}\left(1+T\left(\tilde{a}_{j}\right)\right) a_{j}^{3}}{\sum_{j=1}^{n} \omega_{j}\left(1+T\left(\tilde{a}_{j}\right)\right)}, \frac{\sum_{j=1}^{n} \omega_{j}\left(1+T\left(\tilde{a}_{j}\right)\right) a_{j}^{4}}{\sum_{j=1}^{n} \omega_{j}\left(1+T\left(\tilde{a}_{j}\right)\right)}\right]
\end{aligned}
$$

$\left.1-\left(\prod_{j=1}^{n}\left(1-u_{j}\right)^{\frac{\omega_{j}\left(1+T\left(\tilde{a}_{j}\right)\right)}{\sum_{j=1}^{n} \omega_{j}\left(1+T\left(\tilde{a}_{j}\right)\right)}}\right), \prod_{j=1}^{n} v_{j}^{\frac{\omega_{j}\left(1+T\left(\tilde{a}_{j}\right)\right)}{\sum_{j=1}^{n} \omega_{j}\left(1+T\left(\tilde{a}_{j}\right)\right)}}\right)$

(3) When $\lambda \rightarrow+\infty$, then

$\operatorname{ITFPGWA}\left(\tilde{a}_{1}, \tilde{a}_{2}, \cdots, \tilde{a}_{n}\right)=\max \left(\tilde{a}_{1}, \tilde{a}_{2}, \cdots, \tilde{a}_{n}\right)$. 
Theorem 6. Letting $\operatorname{Sup}\left(\tilde{a}_{i}, \tilde{a}_{j}\right)=k$, for all $i \neq j$, then

$$
\begin{aligned}
& \operatorname{ITFPGWA}\left(\tilde{a}_{1}, \tilde{a}_{2}, \cdots, \tilde{a}_{n}\right) \\
& =\left(\frac{\sum_{j=1}^{n} \omega_{j}\left(1+T\left(\tilde{a}_{j}\right)\right) \tilde{a}_{j}^{\lambda}}{\sum_{j=1}^{n} \omega_{j}\left(1+T\left(\tilde{a}_{j}\right)\right)}\right)^{1 / \lambda}=\left(\sum_{j=1}^{n} \omega_{j} \tilde{a}_{j}^{\lambda}\right)^{1 / \lambda}
\end{aligned}
$$

which indicates that when all the supports are the same, the ITFPGWA operator becomes the generalized weighted average (ITFGWA) operator.

Proof. if $\operatorname{Sup}\left(\tilde{a}_{i}, \tilde{a}_{j}\right)=k$, for all $i \neq j$, then

$$
T\left(\tilde{a}_{j}\right)=\sum_{\substack{i=1 \\ i \neq j}}^{n} \operatorname{Sup}\left(\tilde{a}_{j}, \tilde{a}_{i}\right)=(n-1) k
$$

Thus

$$
\begin{aligned}
& \operatorname{ITFPGWA}\left(\tilde{a}_{1}, \tilde{a}_{2}, \cdots, \tilde{a}_{n}\right)=\left(\frac{\sum_{j=1}^{n} \omega_{j}\left(1+T\left(\tilde{a}_{j}\right)\right) \tilde{a}_{j}^{\lambda}}{\sum_{j=1}^{n} \omega_{j}\left(1+T\left(\tilde{a}_{j}\right)\right)}\right)^{1 / \lambda} \\
& =\left(\frac{\sum_{j=1}^{n} \omega_{j}(1+(n-1) k) \tilde{a}_{j}^{\lambda}}{\sum_{j=1}^{n} \omega_{j}(1+(n-1) k)}\right)^{1 / \lambda}=\left(\frac{\sum_{j=1}^{n} \omega_{j} \tilde{a}_{j}^{\lambda}}{\sum_{j=1}^{n} \omega_{j}}\right)^{1 / \lambda} \\
& =\left(\sum_{j=1}^{n} \omega_{j} \tilde{a}_{j}^{\lambda}\right)^{1 / \lambda}
\end{aligned}
$$

Similar to Theorem 3, 4 and 5, it can be easily proved that the ITFPGWA operator has the following properties:

(1) Theorem 6 (Commutativity).

Let $\tilde{a}_{1}^{\prime}, \tilde{a}_{2}^{\prime}, \cdots, \tilde{a}_{n}^{\prime}$ be any permutation of $\left(\tilde{a}_{1}, \tilde{a}_{2}, \cdots, \tilde{a}_{n}\right)$, then

$\operatorname{ITFPGWA}\left(\tilde{a}_{1}^{\prime}, \tilde{a}_{2}^{\prime}, \cdots, \tilde{a}_{n}^{\prime}\right)=\operatorname{ITFPGWA}\left(\tilde{a}_{1}, \tilde{a}_{2}, \cdots, \tilde{a}_{n}\right)$

(2) Theorem 7 (Idempotency)

Let $\tilde{a}_{j}=\tilde{a}, j=1,2, \cdots, n$, then

$\operatorname{ITFPGWA}\left(\tilde{a}_{1}, \tilde{a}_{2}, \cdots, \tilde{a}_{n}\right)=\tilde{a}$

\section{(3) Theorem8 (Boundedness)}

The ITFPGWA operator lies between the max and min operators:

$$
\begin{aligned}
\min \left(\tilde{a}_{1}, \tilde{a}_{2}, \cdots, \tilde{a}_{n}\right) & \leq \operatorname{ITFPGWA}\left(\tilde{a}_{1}, \tilde{a}_{2}, \cdots, \tilde{a}_{n}\right) \\
& \leq \max \left(\tilde{a}_{1}, \tilde{a}_{2}, \cdots, \tilde{a}_{n}\right)
\end{aligned}
$$

\section{Multi-Attribute Group Decision Making Method Based on the Intuitionistic Trapezoidal Fuzzy Numbers}

\subsection{The description of multiple attribute group decision making problems based on the intuitionistic trapezoidal fuzzy numbers}

For some fuzzy multi-attribute group decision making problems, suppose there are $p$ experts $E=\left\{e_{1}, e_{2}, \cdots, e_{p}\right\}, m$ alternatives $A=\left\{A_{1}, A_{2}, \cdots, A_{m}\right\}$, $n$ decision criteria $C=\left\{C_{1}, C_{2}, \cdots, C_{n}\right\}$, and $\omega=\left(\omega_{1}, \omega_{2}, \cdots, \omega_{n}\right)^{T}$ is the corresponding weight vector of the attributes, where, $\omega_{j} \in[0,1], \sum_{j=1}^{n} \omega_{j}=1$. Let $\underset{\otimes i j}{\tilde{A}^{k}}=\left(\left[a_{i j}^{1 k}, a_{i j}^{2 k}, a_{i j}^{3 k}, a_{i j}^{4 k}\right] ; \mu_{i j}^{k}, v_{i j}^{k}\right)$ be the attribute value in the attribute set $C_{j}$ with respect to the alternative $A_{i}$, given by expert $e_{k}$. where, $\mu_{i j}^{k}$ denotes the extent to which alternative $A_{i}$ belongs to trapezoidal fuzzy number $\left[\left[a_{i j}^{1 k}, a_{i j}^{2 k}, a_{i j}^{3 k}, a_{i j}^{4 k}\right]\right.$ on the criteria $C_{j}, v_{i j}^{k}$ denotes the extent to which alternative $A_{i}$ does not belong to trapezoidal fuzzy number $\left[a_{i j}^{1 k}, a_{i j}^{2 k}, a_{i j}^{3 k}, a_{i j}^{4 k}\right]$ on the criteria $C_{j}$, $0 \leq \mu_{i j}^{k} \leq 1,0 \leq v_{i j}^{k} \leq 1, \mu_{i j}^{k}+v_{i j}^{k} \leq 1 . \gamma=\left(\gamma_{1}, \gamma_{2}, \cdots, \gamma_{p}\right)$ is the expert weight, with $\sum_{k=1}^{p} \gamma_{k}=1$. We can rank the order of the alternatives based on the given information.

Based on these conditions, we can give the steps of the decision making for the fuzzy multi-attribute group decision making problems.

\subsection{Standardize decision matrix}

To eliminate the effect from different physical dimensions to decision results, the decision making 
matrix should be standardized firstly. Suppose that the standardized decision

matrix

is $R^{k}=\left[\tilde{r}_{i j}^{k}\right]_{m \times n}, \tilde{r}_{i j}^{k}=\left(\left[r_{i j}^{1 k}, r_{i j}^{2 k}, r_{i j}^{3 k}, r_{i j}^{4 k}\right] ; \mu_{i j}^{k}, v_{i j}^{k}\right)$,

and to consider two common types of criteria, namely, benefit type and cost type, then the standardized methods are shown as follows:

(1) For cost type of criteria:

$r_{i j}^{l k}=\frac{\max _{j}\left(a_{i j}^{4 k}\right)-a_{i j}^{(5-l) k}}{\max _{j}\left(a_{i j}^{4 k}\right)-\min _{j}\left(a_{i j}^{1 k}\right)} \quad l=1,2,3,4$

(2) For benefit type of criteria:

$$
r_{i j}^{l k}=\frac{a_{i j}^{l k}-\min _{j}\left(a_{i j}^{1 k}\right)}{\max _{j}\left(a_{i j}^{4 k}\right)-\min _{j}\left(a_{i j}^{1 k}\right)} \quad l=1,2,3,4
$$

\subsection{The group decision making approach based on ITFPGWA operator}

Step 1. Calculate the supports.

$\operatorname{Sup}\left(\tilde{r}_{i j}^{k}, \tilde{r}_{i j}^{l}\right)=1-d\left(\tilde{r}_{i j}^{k}, \tilde{r}_{i j}^{l}\right), k, l=1,2, \cdots, p$

which satisfies the support conditions (1)-(3) in Section 3.,where $d\left(\tilde{r}_{i j}^{k}, \tilde{r}_{i j}^{l}\right)$ is Hamming distance between intuitionistic trapezoidal fuzzy numbers $\tilde{r}_{i j}^{k}$ and $\tilde{r}_{i j}^{l}$, which is defined by formula (14).

Step 2. Calculate $T\left(\tilde{r}_{i j}^{k}\right)$

$T\left(\tilde{r}_{i j}^{k}\right)=\sum_{\substack{l=1 \\ l \neq k}}^{p} \operatorname{Sup}\left(\tilde{r}_{i j}^{k}, \tilde{r}_{i j}^{l}\right)$

Step 3. Calculate the weights $w_{i j}^{k}(k=1,2, \cdots, p)$ associated with the intuitionistic trapezoidal fuzzy number $\tilde{r}_{i j}^{k}$

$$
w_{i j}^{k}=\frac{\gamma_{k}\left(1+T\left(\tilde{r}_{i j}^{k}\right)\right)}{\sum_{k=1}^{p} \gamma_{k}\left(1+T\left(\tilde{r}_{i j}^{k}\right)\right)}
$$

Step 4. Aggregate the evaluation information of each expert by ITFPGWA operator

$$
\begin{aligned}
& \tilde{r}_{i j}=\operatorname{ITFPGWA}\left(\tilde{r}_{i j}^{1}, \tilde{r}_{i j}^{2}, \cdots, \tilde{r}_{i j}^{p}\right) \\
& =\left(\left[\left(\sum_{k=1}^{p} w_{i j}^{k} r_{i j}^{k \lambda \lambda}\right)^{1 / \lambda},\left(\sum_{k=1}^{p} w_{i j}^{k} r_{i j}^{2 k \lambda}\right)^{1 / \lambda},\left(\sum_{k=1}^{p} w_{i j}^{k} r_{i j}^{3 k \lambda}\right)^{1 / \lambda},\right.\right.
\end{aligned}
$$

$$
\begin{aligned}
& \left.\left(\sum_{k=1}^{p} w_{i j}^{k} r_{i j}^{4 k \lambda}\right)^{1 / \lambda}\right] ;\left(1-\left(\prod_{k=1}^{p}\left(1-u_{i j}^{k \lambda}\right)^{w_{i j}^{k}}\right)\right)^{1 / \lambda}, \\
& \left.1-\left(1-\prod_{k=1}^{p}\left(1-\left(1-v_{i j}^{k}\right)^{\lambda}\right)^{w_{i j}^{k}}\right)^{1 / \lambda}\right)
\end{aligned}
$$

Step 5. Calculate $T\left(\tilde{r}_{i j}\right)$

$$
T\left(\tilde{r}_{i j}\right)=\sum_{\substack{l=1 \\ l \neq j}}^{n} \operatorname{Sup}\left(\tilde{r}_{i j}, \tilde{r}_{i l}\right)
$$

Step6. Calculate the weights $\varpi_{i j}(j=1,2, \cdots, n)$ associated with the intuitionistic trapezoidal fuzzy number $\tilde{r}_{i j}$

$$
\varpi_{i j}=\frac{\omega_{j}\left(1+T\left(\tilde{r}_{i j}\right)\right)}{\sum_{j=1}^{n} \omega_{j}\left(1+T\left(\tilde{r}_{i j}\right)\right)}
$$

Step 7. Calculate the comprehensive evaluation value of each alternative

$$
\begin{aligned}
\tilde{z}_{i}= & \operatorname{ITFPGWA}\left(\tilde{r}_{i 1}, \tilde{r}_{i 2}, \cdots, \tilde{r}_{i n}\right) \\
= & \left(\left[\left(\sum_{j=1}^{n} \varpi_{i j} r_{i j}^{1 \lambda}\right)^{1 / \lambda},\left(\sum_{j=1}^{n} \varpi_{i j} r_{i j}^{2 \lambda}\right)^{1 / \lambda},\left(\sum_{j=1}^{n} \varpi_{i j} r_{i j}^{3 \lambda}\right)^{1 / \lambda},\right.\right. \\
& \left.\left(\sum_{j=1}^{n} \varpi_{i j} r_{i j}^{4 \lambda}\right)^{1 / \lambda}\right] ;\left(1-\left(\prod_{j=1}^{n}\left(1-u_{i j}^{\lambda}\right)^{\sigma_{i j}}\right)\right)^{1 / \lambda}, \\
& \left.1-\left(1-\prod_{n=1}^{n}\left(1-\left(1-v_{i j}\right)^{\lambda}\right)^{\sigma_{i j}}\right)^{1 / \lambda}\right)
\end{aligned}
$$

Step 8. Rank $\tilde{z}_{i}(i=1,2, \cdots, m)$ in descending order by using the ranking method of intuitionistic trapezoidal fuzzy number described in Definition 6.

Step 9. Rank all the alternatives and select the best one(s) in accordance with the ranking of $\tilde{z}_{i}(i=1,2, \cdots, m)$.

Step 10. End. 


\section{Example}

A practical use of the proposed approach involves the evaluating the technological innovation ability of the four enterprises $\left\{A_{1}, A_{2}, A_{3}, A_{4}\right\}$, the evaluating attributes have the ability of innovative resources investment $\left(C_{1}\right)$, the ability of innovation management $\left(C_{2}\right)$, the ability of innovation tendency $\left(C_{3}\right)$ and the ability of research and development $\left(C_{4}\right)$. Based on the four attributes, the three experts $\left\{e_{1}, e_{2}, e_{3}\right\}$ evaluated the technological innovation ability of the four enterprises. Supposed that $\gamma=(0.4,0.32,0.28)$ is the expert weight vector, and $\omega=(0.3,0.2,0.2,0.3)$ is attribute weight vector. The attribute values given by the experts take the form of intuitionistic trapezoidal fuzzy numbers, shown in Tables 1, 2 and 3. The problem is ranking the four enterprises based on their technological innovation ability.

Table 1 Attribute values of alternatives given by

\begin{tabular}{ccc}
\multicolumn{3}{c}{ expert $e_{1}$} \\
\hline \multicolumn{3}{c}{$C_{1}$} \\
\hline$A_{1}$ & $([0.2,0.2,0.3,0.3] ; 0.7,0.3)$ & $([0.4,0.4,0.5,0.5] ; 0.7,0.3)$ \\
$A_{2}$ & $([0.4,0.5,0.6,0.7] ; 0.6,0.3)$ & $([0.6,0.7,0.8,0.9] ; 0.8,0.1)$ \\
$A_{3}$ & $([0.3,0.3,0.3,0.3] ; 0.6,0.3)$ & $([0.4,0.6,0.7,0.8] ; 0.6,0.3)$ \\
$A_{4}$ & $([0.1,0.2,0.3,0.4] ; 0.6,0.2)$ & $([0.3,0.4,0.5,0.5] ; 0.8,0.2)$ \\
\hline
\end{tabular}

Table 1 Attribute values of alternatives given by expert $e_{1}$ (Cont.)

\begin{tabular}{ccc}
\hline & $C_{3}$ & $C_{4}$ \\
\hline$A_{1}$ & $([0.4,0.5,0.6,0.7] ; 0.7,0.3)$ & $([0.5,0.6,0.6,0.7] ; 0.7,0.2)$ \\
$A_{2}$ & $([0.5,0.6,0.6,0.7] ; 0.8,0.2)$ & $([0.3,0.4,0.5,0.6] ; 0.7,0.3)$ \\
$A_{3}$ & $([0.3,0.4,0.5,0.6] ; 0.5,0.5)$ & $([0.4,0.5,0.6,0.7] ; 0.8,0.1)$ \\
$A_{4}$ & $([0.2,0.3,0.5,0.6] ; 0.6,0.4)$ & $([0.2,0.3,0.3,0.4] ; 0.6,0.3)$ \\
\hline
\end{tabular}

Table 2 Attribute values of alternatives given by

\begin{tabular}{ccc}
\multicolumn{3}{c}{$C_{1}$ expert $e_{2}$} \\
\hline \multicolumn{3}{c}{$C_{2}$} \\
\hline$A_{1}$ & $([0.2,0.3,0.3,0.4] ; 0.7,0.3)$ & $([0.4,0.4,0.4,0.4] ; 0.7,0.3)$ \\
$A_{2}$ & $([0.3,0.4,0.5,0.6] ; 0.6,0.3)$ & $([0.3,0.4,0.5,0.6] ; 0.5,0.5)$ \\
$A_{3}$ & $([0.4,0.4,0.5,0.5] ; 0.8,0.2)$ & $([0.2,0.3,0.5,0.6] ; 0.6,0.4)$ \\
$A_{4}$ & $([0.2,0.2,0.3,0.3] ; 0.9,0.0)$ & $([0.3,0.4,0.5,0.6] ; 0.8,0.2)$ \\
\hline
\end{tabular}

Table 2 Attribute values of alternatives given by expert $e_{2}$ (Cont.)

\begin{tabular}{ccc}
\hline & $C_{3}$ & $C_{4}$ \\
\hline$A_{1}$ & $([0.4,0.5,0.6,0.7] ; 0.6,0.3)$ & $([0.4,0.5,0.6,0.7] ; 0.8,0.0)$ \\
$A_{2}$ & $([0.4,0.5,0.5,0.6] ; 0.8,0.1)$ & $([0.5,0.6,0.7,0.8] ; 0.8,0.2)$ \\
$A_{3}$ & $([0.3,0.4,0.5,0.7] ; 0.6,0.3)$ & $([0.4,0.4,0.4,0.4] ; 0.6,0.3)$ \\
$A_{4}$ & $([0.1,0.2,0.3,0.4] ; 0.8,0.1)$ & $([0.3,0.4,0.4,0.5] ; 0.7,0.0)$ \\
\hline
\end{tabular}

Table 3 Attribute values of alternatives given by expert $e_{3}$

\begin{tabular}{ccc}
\hline & $C_{1}$ & $C_{2}$ \\
\hline$A_{1}$ & $([0.3,0.3,0.4,0.4] ; 0.7,0.3)$ & $([0.4,0.4,0.6,0.6] ; 0.6,0.3)$ \\
$A_{2}$ & $([0.2,0.3,0.4,0.5] ; 0.6,0.3)$ & $([0.5,0.5,0.5,0.5] ; 0.7,0.1)$ \\
$A_{3}$ & $([0.2,0.3,0.4,0.6] ; 0.6,0.2)$ & $([0.5,0.6,0.7,0.8] ; 0.8,0.2)$ \\
$A_{4}$ & $([0.2,0.3,0.4,0.5] ; 0.8,0.2)$ & $([0.3,0.3,0.4,0.4] ; 0.8,0.0)$ \\
\hline
\end{tabular}

Table 3 Attribute values of alternatives given by

\begin{tabular}{ccc}
\multicolumn{3}{c}{ expert $e_{3}$ (Cont.) } \\
\hline \multicolumn{3}{c}{$C_{3}$} \\
\hline$A_{1}$ & $([0.4,0.5,0.7,0.8] ; 0.7,0.3)$ & $([0.5,0.6,0.6,0.7] ; 0.8,0.0)$ \\
$A_{2}$ & $([0.4,0.4,0.5,0.5] ; 0.7,0.3)$ & $([0.6,0.7,0.7,0.8] ; 0.6,0.3)$ \\
$A_{3}$ & $([0.3,0.4,0.4,0.5] ; 0.6,0.3)$ & $([0.4,0.6,0.7,0.8] ; 0.6,0.3)$ \\
$A_{4}$ & $([0.2,0.2,0.3,0.3] ; 0.8,0.1)$ & $([0.2,0.3,0.3,0.4] ; 0.8,0.0)$ \\
\hline
\end{tabular}




\subsection{Steps using the proposed method are shown as follows}

(1) Standardize decision matrix

The standardized decision matrix $R^{k}=\left[\tilde{r}_{i j}^{k}\right]_{4 \times 4}$, $k=1,2,3$ produced by formula (23) are shown as follows

$$
\begin{aligned}
R^{1}= & \begin{array}{l}
([0.167,0.167,0.333,0.333] ; 0.700,0.300) \\
([0.500,0.667,0.833,1.000] ; 0.600,0.300) \\
([0.333,0.333,0.333,0.333] ; 0.600,0.300) \\
([0.000,0.167,0.333,0.500] ; 0.600,0.200)
\end{array} \\
& ([0.167,0.167,0.333,0.333] ; 0.700,0.300) \\
& ([0.500,0.667,0.833,1.000] ; 0.800,0.100) \\
& ([0.167,0.500,0.667,0.833] ; 0.600,0.300) \\
& ([0.000,0.167,0.333,0.333] ; 0.800,0.200) \\
& ([0.400,0.600,0.800,1.000] ; 0.700,0.300)
\end{aligned}
$$$$
\text { ([0.600,0.800,0.800,1.000];0.800,0.200) }
$$$$
\text { ([0.200,0.400,0.600,0.800];0.500,0.500) }
$$$$
([0.000,0.200,0.600,0.800] ; 0.600,0.400)
$$$$
\text { ([0.600,0.800,0.8001.000];0.700,0.200) }
$$$$
\text { ([0.200,0.400,0.6000.800];0.700,0.300) }
$$$$
\text { ([0.400,0.600,0.8001.000];0.800,0.100) }
$$$$
\text { ([0.000,0.200,0.2000.400];0.600,0.300) }
$$$$
R^{2}=\left[\begin{array}{l}
([0.000,0.250,0.250,0.500] ; 0.700,0.300) \\
([0.250,0.500,0.750,1.000] ; 0.600,0.300) \\
([0.500,0.500,0.750,0.750] ; 0.800,0.200) \\
([0.000,0.000,0.250,0.250] ; 0.900,0.000)
\end{array}\right.
$$$$
\text { ([0.500,0.500,0.500,0.500];0.700,0.300) }
$$$$
\text { ([0.250,0.500,0.750,1.000];0.500,0.500) }
$$$$
\text { ([0.000,0.250,0.750,1.000];0.600,0.400) }
$$$$
\text { ([0.250,0.500,0.750,1.000];0.800,0.200) }
$$$$
\text { ([0.500,0.667,0.833,1.000];0.600,0.300) }
$$$$
([0.500,0.667,0.667,0.833] ; 0.800,0.100)
$$$$
([0.333,0.500,0.667,1.000] ; 0.600,0.300)
$$$$
\text { ([0.000,0.167,0.333,0.500];0.800,0.100) }
$$$$
([0.200,0.400,0.6000 .800] ; 0.800,0.000)]
$$$$
\text { ([0.400,0.600,0.8001.000];0.800,0.200) }
$$$$
\text { ([0.200,0.200,0.2000.200];0.600,0.300) }
$$$$
([0.000,0.200,0.2000 .400] ; 0.700,0.000)]
$$

$$
R^{3}=\left[\begin{array}{l}
([0.250,0.250,0.500,0.500] ; 0.700,0.300) \\
([0.000,0.250,0.500,0.750] ; 0.600,0.300) \\
([0.000,0.250,0.500,1.000] ; 0.600,0.200) \\
([0.000,0.250,0.500,0.750] ; 0.800,0.200)
\end{array}\right.
$$

([0.200,0.200,0.600,0.600];0.600,0.300)

([0.400,0.400,0.400,0.400];0.700,0.100)

([0.400,0.600,0.800,1.000];0.800,0.200)

([0.000,0.000,0.200,0.200];0.800,0.000) ([0.333,0.500,0.833,1.000];0.700,0.300)

([0.333,0.333,0.500,0.500];0.700,0.300)

([0.167,0.333,0.333,0.500];0.600,0.300)

([0.000,0.000,0.167,0.167];0.800,0.100)

([0.500,0.667,0.6670.833];0.800,0.000)

([0.667,0.833,0.8331.000];0.600,0.300)

([0.333,0.667,0.8331.000];0.600,0.300)

([0.000,0.167,0.1670.333];0.800,0.000)

(2) Calculate the supports $\operatorname{Sup}\left(\tilde{r}_{i j}^{k}, \tilde{r}_{i j}^{l}\right) \quad k, l=1,2,3 . \quad i, j=1,2,3,4 . \quad$ by formula (24) (for simplicity, we denote $\operatorname{Sup}\left(\tilde{r}_{i j}^{k}, \tilde{r}_{i j}^{l}\right)$ with $S_{i j}^{k l}$

$$
\begin{aligned}
& S_{11}^{12}=S_{11}^{13}=S_{11}^{21}=S_{11}^{23}=S_{11}^{31}=S_{11}^{32}=0.913 \\
& S_{12}^{12}=S_{12}^{12}=0.825, S_{12}^{13}=S_{12}^{31}=0.915, S_{12}^{23}=S_{12}^{32}=0.870 \\
& S_{13}^{12}=S_{13}^{21}=0.968, S_{13}^{13}=S_{13}^{31}=0.965, S_{13}^{23}=S_{13}^{32}=0.933 \\
& S_{14}^{12}=S_{14}^{21}=0.850, S_{14}^{13}=S_{14}^{31}=1.000, S_{14}^{23}=S_{14}^{32}=0.850 \\
& S_{21}^{12}=S_{21}^{21}=0.919, S_{21}^{13}=S_{21}^{31}=0.756, S_{21}^{23}=S_{21}^{32}=0.838 \\
& S_{22}^{12}=S_{22}^{21}=0.675, S_{22}^{13}=S_{22}^{31}=0.683, S_{22}^{23}=S_{22}^{32}=0.875 \\
& S_{23}^{12}=S_{23}^{21}=0.927, S_{23}^{13}=S_{23}^{31}=0.652, S_{23}^{23}=S_{23}^{32}=0.725 \\
& S_{22}^{12}=S_{24}^{21}=0.790, S_{24}^{13}=S_{24}^{31}=0.808, S_{24}^{23}=S_{24}^{32}=0.894 \\
& S_{31}^{12}=S_{31}^{21}=0.717, S_{31}^{13}=S_{31}^{31}=0.781, S_{31}^{23}=S_{31}^{32}=0.756 \\
& S_{32}^{12}=S_{32}^{21}=0.910, S_{32}^{13}=S_{32}^{31}=0.792, S_{32}^{23}=S_{32}^{32}=0.740 \\
& S_{33}^{12}=S_{33}^{21}=0.844, S_{33}^{13}=S_{33}^{31}=0.954, S_{33}^{23}=S_{33}^{32}=0.810 \\
& S_{34}^{12}=S_{34}^{21}=0.535, S_{34}^{13}=S_{34}^{31}=0.865, S_{34}^{23}=S_{34}^{32}=0.670 \\
& S_{41}^{12}=S_{41}^{21}=0.942, S_{41}^{13}=S_{41}^{31}=0.875, S_{41}^{23}=S_{41}^{32}=0.819 \\
& S_{42}^{12}=S_{42}^{21}=0.667, S_{42}^{13}=S_{42}^{31}=0.923, S_{42}^{23}=S_{42}^{32}=0.590 \\
& S_{43}^{12}=S_{43}^{21}=0.962, S_{43}^{13}=S_{43}^{31}=0.831, S_{43}^{23}=S_{43}^{32}=0.858
\end{aligned}
$$


$S_{44}^{12}=S_{44}^{21}=0.960, S_{44}^{13}=S_{44}^{31}=0.980, S_{44}^{23}=S_{44}^{32}=0.980$

(3) Calculate $T\left(\tilde{r}_{i j}^{k}\right) \quad i, j=1,2,3,4 . \quad k=1,2,3$. by formula (25)

$$
\begin{aligned}
& T\left(\tilde{r}_{11}^{1}\right)=T\left(\tilde{r}_{11}^{2}\right)=T\left(\tilde{r}_{11}^{3}\right)=1.825, \\
& T\left(\tilde{r}_{12}^{1}\right)=1.740, T\left(\tilde{r}_{12}^{2}\right)=1.695, T\left(\tilde{r}_{12}^{3}\right)=1.785 \\
& T\left(\tilde{r}_{13}^{1}\right)=1.933, T\left(\tilde{r}_{13}^{2}\right)=1.902, T\left(\tilde{r}_{13}^{3}\right)=1.898, \\
& T\left(\tilde{r}_{14}^{1}\right)=1.850, T\left(\tilde{r}_{14}^{2}\right)=1.700, T\left(\tilde{r}_{14}^{3}\right)=1.850 \\
& T\left(\tilde{r}_{21}^{1}\right)=1.675, T\left(\tilde{r}_{21}^{2}\right)=1.756, T\left(\tilde{r}_{21}^{3}\right)=1.594, \\
& T\left(\tilde{r}_{22}^{1}\right)=1.358, T\left(\tilde{r}_{22}^{2}\right)=1.550, T\left(\tilde{r}_{22}^{3}\right)=1.558 \\
& T\left(\tilde{r}_{23}^{1}\right)=1.578, T\left(\tilde{r}_{23}^{2}\right)=1.652, T\left(\tilde{r}_{23}^{3}\right)=1.377, \\
& T\left(\tilde{r}_{24}^{1}\right)=1.598, T\left(\tilde{r}_{24}^{2}\right)=1.684, T\left(\tilde{r}_{24}^{3}\right)=1.703 \\
& T\left(\tilde{r}_{31}^{1}\right)=1.498, T\left(\tilde{r}_{31}^{2}\right)=1.473, T\left(\tilde{r}_{31}^{3}\right)=1.538, \\
& T\left(\tilde{r}_{32}^{1}\right)=1.703, T\left(\tilde{r}_{32}^{2}\right)=1.650, T\left(\tilde{r}_{32}^{3}\right)=1.532 \\
& T\left(\tilde{r}_{33}^{1}\right)=1.798, T\left(\tilde{r}_{33}^{2}\right)=1.654, T\left(\tilde{r}_{33}^{3}\right)=1.765, \\
& T\left(\tilde{r}_{34}^{1}\right)=1.400, T\left(\tilde{r}_{34}^{2}\right)=1.205, T\left(\tilde{r}_{34}^{3}\right)=1.535 \\
& T\left(\tilde{r}_{41}^{1}\right)=1.817, T\left(\tilde{r}_{41}^{2}\right)=1.760, T\left(\tilde{r}_{41}^{3}\right)=1.694, \\
& T\left(\tilde{r}_{42}^{1}\right)=1.590, T\left(\tilde{r}_{42}^{2}\right)=1.257, T\left(\tilde{r}_{42}^{3}\right)=1.513 \\
& T\left(\tilde{r}_{43}^{1}\right)=1.793, T\left(\tilde{r}_{43}^{2}\right)=1.820, T\left(\tilde{r}_{43}^{3}\right)=1.689, \\
& T\left(\tilde{r}_{44}^{1}\right)=1.940, T\left(\tilde{r}_{44}^{2}\right)=1.940, T\left(\tilde{r}_{44}^{3}\right)=1.960
\end{aligned}
$$

$$
\text { Calculate }
$$

the weights

$w_{i j}^{k} \quad(i, j=1,2,3,4 . \quad k=1,2,3)$ by formula (26) $w_{11}^{1}=0.400, w_{11}^{2}=0.320, w_{11}^{3}=0.280$, $w_{12}^{1}=0.400, w_{12}^{2}=0.315, w_{12}^{3}=0.285$ $w_{13}^{1}=0.403, w_{13}^{2}=0.319, w_{13}^{3}=0.279$, $w_{14}^{1}=0.407, w_{14}^{2}=0.308, w_{14}^{3}=0.285$ $w_{21}^{1}=0.400, w_{21}^{2}=0.329, w_{21}^{3}=0.271$, $w_{22}^{1}=0.381, w_{22}^{2}=0.330, w_{22}^{3}=0.289$ $w_{23}^{1}=0.405, w_{23}^{2}=0.333, w_{23}^{3}=0.261$, $w_{24}^{1}=0.391, w_{24}^{2}=0.324, w_{24}^{3}=0.285$ $w_{31}^{1}=0.400, w_{31}^{2}=0.316, w_{31}^{3}=0.284$, $w_{32}^{1}=0.410, w_{32}^{2}=0.321, w_{32}^{3}=0.269$ $w_{33}^{1}=0.408, w_{33}^{2}=0.310, w_{33}^{3}=0.282$,

$$
\begin{aligned}
& w_{34}^{1}=0.404, w_{34}^{2}=0.297, w_{34}^{3}=0.299 \\
& w_{41}^{1}=0.408, w_{41}^{2}=0.320, w_{41}^{3}=0.273, \\
& w_{42}^{1}=0.421, w_{42}^{2}=0.293, w_{42}^{3}=0.286 \\
& w_{43}^{1}=0.403, w_{43}^{2}=0.325, w_{43}^{3}=0.272, \\
& w_{44}^{1}=0.399, w_{44}^{2}=0.319, w_{44}^{3}=0.281
\end{aligned}
$$

(5) Aggregate the evaluation information of each expert by ITFPGWA operator, suppose $\lambda=1$

$R=\left[\begin{array}{l}([0.137,0.217,0.353,0.433] ; 0.700,0.300) \\ ([0.282,0.499,0.716,0.932] ; 0.600,0.300) \\ ([0.291,0.362,0.513,0.655] ; 0.679,0.235) \\ ([0.000,0.136,0.352,0.488] ; 0.787,0.000)\end{array}\right.$ $([0.281,0.281,0.462,0.462] ; 0.674,0.300)$ $([0.389,0.535,0.680,0.826] ; 0.696,0.170)$ $([0.176,0.447,0.729,0.932] ; 0.668,0.295)$ $([0.073,0.217,0.417,0.491] ; 0.800,0.000)$ $([0.413,0.593,0.820,1.000] ; 0.671,0.300)$ $([0.497,0.634,0.677,0.814] ; 0.778,0.176)$ $([0.232,0.412,0.545,0.777] ; 0.562,0.370)$ $([0.000,0.135,0.396,0.530] ; 0.736,0.175)$ $([0.448,0.639,0.700,0.891] ; 0.764,0.000)]$ $([0.398,0.588,0.731,0.922] ; 0.714,0.263)$ $([0.321,0.501,0.632,0.762] ; 0.698,0.192)$ $([0.000,0.191,0.191,0.381] ; 0.700,0.000)]$

(6) Calculate $T\left(\tilde{r}_{i j}\right) \quad(i, j=1,2,3,4)$ by formula (28)

$$
\begin{gathered}
T\left(\tilde{r}_{11}\right)=2.268, T\left(\tilde{r}_{12}\right)=2.380, \\
T\left(\tilde{r}_{13}\right)=2.380, T\left(\tilde{r}_{14}\right)=2.168 \\
T\left(\tilde{r}_{21}\right)=2.717, T\left(\tilde{r}_{22}\right)=2.851, \\
T\left(\tilde{r}_{23}\right)=2.754, T\left(\tilde{r}_{24}\right)=2.842 \\
T\left(\tilde{r}_{31}\right)=2.768, T\left(\tilde{r}_{32}\right)=2.714, \\
T\left(\tilde{r}_{33}\right)=2.733, T\left(\tilde{r}_{34}\right)=2.717 \\
T\left(\tilde{r}_{41}\right)=2.861, T\left(\tilde{r}_{42}\right)=2.778, \\
T\left(\tilde{r}_{43}\right)=2.853, T\left(\tilde{r}_{44}\right)=2.743
\end{gathered}
$$

(7) Calculate the weights $\varpi_{i j}(i, j=1,2,3,4)$ by formula (29)

$\varpi_{11}=0.299, \varpi_{12}=0.206, \varpi_{13}=0.206, \varpi_{14}=0.289$ 


$$
\begin{aligned}
& \varpi_{21}=0.294, \varpi_{22}=0.203, \varpi_{23}=0.198, \varpi_{24}=0.304 \\
& \varpi_{31}=0.303, \varpi_{32}=0.199, \varpi_{33}=0.200, \varpi_{34}=0.299 \\
& \varpi_{41}=0.304, \varpi_{42}=0.198, \varpi_{43}=0.202, \varpi_{44}=0.295
\end{aligned}
$$

(8) Calculate the comprehensive evaluation value of each alternative by formula (30), suppose $\lambda=1$

$\tilde{z}_{1}=([0.314,0.430,0.572,0.688] ; 0.710,0.000)$

$\tilde{z}_{2}=([0.382,0.560,0.706,0.884] ; 0.696,0.231)$

$\tilde{z}_{3}=([0.265,0.430,0.598,0.766] ; 0.662,0.254)$

$\tilde{z}_{4}=([0.015,0.168,0.326,0.466] ; 0.757,0.000)$

(9) Calculate expected value $\tilde{z}_{i}(i=1,2,3,4)$ by formula (13)

$$
\begin{aligned}
& I\left(\tilde{z}_{1}\right)=0.428, I\left(\tilde{z}_{2}\right)=0.463, \\
& I\left(\tilde{z}_{3}\right)=0.363, I\left(\tilde{z}_{4}\right)=0.214
\end{aligned}
$$

(10) Rank the alternatives

According to the expected value, the ranking is $A_{2} \succ A_{1} \succ A_{3} \succ A_{4}$.

\subsection{Discussion}

In order to illustrate the influence of the parameter $\lambda$ on

\begin{tabular}{|c|c|c|}
\hline$\lambda$ & Expected values $\tilde{z}_{i}(i=1,2,3,4)$ & Ranking \\
\hline$\lambda \rightarrow 0$ & $\begin{array}{l}I\left(\tilde{z}_{1}\right)=0.328, I\left(\tilde{z}_{2}\right)=0.379 \\
I\left(\tilde{z}_{3}\right)=0.287, I\left(\tilde{z}_{4}\right)=0.155\end{array}$ & $A_{2} \succ A_{1} \succ A_{3} \succ A_{4}$ \\
\hline$\lambda=0.1$ & $\begin{array}{l}I\left(\tilde{z}_{1}\right)=0.356, I\left(\tilde{z}_{2}\right)=0.412 \\
I\left(\tilde{z}_{3}\right)=0.300, I\left(\tilde{z}_{4}\right)=0.161\end{array}$ & $A_{2} \succ A_{1} \succ A_{3} \succ A_{4}$ \\
\hline$\lambda=0.5$ & $\begin{array}{l}I\left(\tilde{z}_{1}\right)=0.402, I\left(\tilde{z}_{2}\right)=0.448 \\
I\left(\tilde{z}_{3}\right)=0.341, I\left(\tilde{z}_{4}\right)=0.193\end{array}$ & $A_{2} \succ A_{1} \succ A_{3} \succ A_{4}$ \\
\hline$\lambda=1.0$ & $\begin{array}{l}I\left(\tilde{z}_{1}\right)=0.428, I\left(\tilde{z}_{2}\right)=0.463 \\
I\left(\tilde{z}_{3}\right)=0.363, I\left(\tilde{z}_{4}\right)=0.214\end{array}$ & $A_{2} \succ A_{1} \succ A_{3} \succ A_{4}$ \\
\hline$\lambda=1.5$ & $\begin{array}{l}I\left(\tilde{z}_{1}\right)=0.450, I\left(\tilde{z}_{2}\right)=0.475 \\
I\left(\tilde{z}_{3}\right)=0.379, I\left(\tilde{z}_{4}\right)=0.233\end{array}$ & $A_{2} \succ A_{1} \succ A_{3} \succ A_{4}$ \\
\hline$\lambda=2.0$ & $\begin{array}{l}I\left(\tilde{z}_{1}\right)=0.469, I\left(\tilde{z}_{2}\right)=0.484 \\
I\left(\tilde{z}_{3}\right)=0.392, I\left(\tilde{z}_{4}\right)=0.252\end{array}$ & $A_{2} \succ A_{1} \succ A_{3} \succ A_{4}$ \\
\hline$\lambda=5.0$ & $\begin{array}{l}I\left(\tilde{z}_{1}\right)=0.544, I\left(\tilde{z}_{2}\right)=0.527 \\
I\left(\tilde{z}_{3}\right)=0.444, I\left(\tilde{z}_{4}\right)=0.343\end{array}$ & $A_{1} \succ A_{2} \succ A_{3} \succ A_{4}$ \\
\hline$\lambda=10.0$ & $\begin{array}{l}I\left(\tilde{z}_{1}\right)=0.601, I\left(\tilde{z}_{2}\right)=0.572 \\
I\left(\tilde{z}_{3}\right)=0.492, I\left(\tilde{z}_{4}\right)=0.425\end{array}$ & $A_{1} \succ A_{2} \succ A_{3} \succ A_{4}$ \\
\hline$\lambda=100.0$ & $\begin{array}{l}I\left(\tilde{z}_{1}\right)=0.709, I\left(\tilde{z}_{2}\right)=0.688 \\
I\left(\tilde{z}_{3}\right)=0.615, I\left(\tilde{z}_{4}\right)=0.571\end{array}$ & $A_{1} \succ A_{2} \succ A_{3} \succ A_{4}$ \\
\hline
\end{tabular}
decision making of this example, we use the different value $\lambda$ in step (5) and (8) to rank the alternatives. The ranking results are shown in Table 4 .

Table 4 Ordering of the alternatives by utilizing the different $\lambda$ in ITFPGWA operator

As we can see from Table 4, the ordering of the alternatives may be different for the different value $\lambda$ in 
ITFPGWA operator. Thus, the organization can properly select the desirable alternative according to his interest and the actual needs.

In addition, in order to verify the validity of the method proposed in this paper, we adopt the same the method proposed by Wang and Zhang ${ }^{15}$, and re-rank the alternatives .The ranking result is shown as follows: $A_{2} \succ A_{1} \succ A_{3} \succ A_{4}$.

Obviously, two methods have the same ranking results; this verifies the validity of the method in this paper.

\section{Conclusion}

In this paper, with respect to multiple attribute decision making (MADM) problems in which the attribute value takes the form of intuitionistic trapezoidal fuzzy number, a new decision making analysis method is developed. Firstly, some operational laws and expected values of intuitionistic trapezoidal fuzzy numbers, and distance between two intuitionistic trapezoidal fuzzy numbers, are introduced, and the comparison method for the intuitionistic trapezoidal fuzzy numbers is proposed. Then, combined the power aggregation operator and the generalized aggregation operators, we propose a power generalized average (PGA) operator, and study some properties of the PGA operator, such as idempotency, boundary, commutativity, etc. At the same time, we analyze some special cases of the generalized parameters in PGA operator. Based on the support measure, the weights associated with PGA operator are derived directly from the aggregated intuitionistic trapezoidal fuzzy information, and are in accordance with the principle that the more support (or closer) the intuitionistic trapezoidal fuzzy to all the other values, the more the weight. Therefore, the developed operator can relieve the influence of unfair arguments on the aggregated results, and thus can make the aggregated results more reasonable. Furthermore, we also propose an intuitionistic trapezoidal fuzzy power generalized weighted average (ITFPGWA) operator for the intuitionistic trapezoidal fuzzy information, and give some properties of the ITFPGWA operator and an approach to deal with group decision making problems under intuitionistic trapezoidal fuzzy information based on the ITFPGWA operator. The prominent characteristic of the developed approach is that they can take all the decision arguments and their relationships into account. Finally, an illustrative example is given to illustrate the decision-making steps, to verify the developed approach and to demonstrate its practicality and effectiveness. In the future, we shall continue working in the extension and application of the developed method to other domains.

\section{Acknowledgment}

This paper is supported by the National Natural Science Foundation of China (No. 71271124), the Humanities and Social Sciences Research Project of Ministry of Education of China (No. 13YJC630104 and No. 09YJA630088), the Natural Science Foundation of Shandong Province (No.ZR2011FM036), Shandong Provincial Social Science Planning Project (No.13BGLJ10) and Graduate Education Innovation Projects of Shandong Province (No.SDYY12065). The author also would like to express appreciation to the anonymous reviewers and Editors for their very helpful comments that improved the paper.

\section{References}

1. L. A. Zadeh, Fuzzy sets, Information and Control 8(1965) 338- 356.

2. K.T. Atanassov, Intuitionistic fuzzy sets, Fuzzy Sets and Systems 20(1986) 87-96.

3. K.T. Atanassov, More on intuitionistic fuzzy sets, Fuzzy Sets and Systems 33(1989) 37-46.

4. Z.S. Xu, R.R. Yager, Some geometric aggregation operators based on intuitionistic fuzzy sets, International Journal of General Systems 35(2006) 417-433.

5. Z.S. Xu, Intuitionistic fuzzy aggregation operators, IEEE Transactions on Fuzzy Systems 15(2007) 1179-1187.

6. K.T. Atanassov, G. Gargov, interval-valued intuitionistic fuzzy sets, Fuzzy Sets and Systems 3(1989) 343-349.

7. K.T. Atanassov, Operators over interval-valued intuitionistic fuzzy sets, Fuzzy Sets and Systems 64(1994) 159-174.

8. Z.S. Xu, Methods for aggregating interval-valued intuitionistic fuzzy information and their application to decision making, Control and Decision 22(2007) 215219.

9. Z.S. Xu, J. Chen, An approach to group decision making based on interval-valued intuitionistic judgment matrices, Systems Engineering Theory and Practice 27(2007) 26133.

10. M.H. Shu, C.H. Cheng, J.R. Chang, Using intuitionistic fuzzy sets for fault-tree analysis on printed circuit board 
assembly, Microelectronics Reliability 46(2006) 21392148.

11. X. Zhang, P.D. Liu, Method for aggregating triangular intuitionistic fuzzy information and its application to decision making, Technological and Economic Development of Economy 16(2010) 280-290.

12. J.Q. Wang, Overview on fuzzy multi-criteria decisionmaking approach, Control and Decision 23(2008) 601606.

13. J.Q. Wang, Z.H. Zhang, Programming method of multicriteria decision-making based on intuitionistic fuzzy number with incomplete certain information, Control and Decision 23(2008) 1145-1148.

14. J.Q. Wang, Z.H. Zhang, Multi-criteria decision-making method with incomplete certain information based on intuitionistic fuzzy number, Control and Decision 24(2009) 226-230.

15. J.Q. Wang, Z.H. Zhang, Aggregation operators on intuitionistic trapezoidal fuzzy number and its application to multi-criteria decision making problems, Journal of Systems Engineering and Electronics 20(2009) 321-326.

16. S.P. Wan, J.Y. Dong, Method of intuitionistic trapezoidal fuzzy number for multi-attribute group decision, Control and Decision 25(2010) 773-776.

17. G.W. Wei, Some Arithmetic Aggregation Operators with Intuitionistic Trapezoidal Fuzzy Numbers and Their Application to Group Decision Making, Journal of Computers 5(2010) 345-351.

18. R.R. Yager, Generalized OWA aggregation operators, Fuzzy Optimization and Decisio n Making 3(2004) 93107.

19. D.F. Li, Multiattribute decision making method based on generalized OWA operators with intuitionistic fuzzy sets, Expert Systems with Applications 37(2010) 8673-8678.

20. H. Zhao, Z.S. Xu, M. Ni, S. Liu, Generalized aggregation operators for intuitionistic fuzzy sets, International Journal of Intelligent Systems 25(2010) 1-30.

21. J.M. Merigó, M. Casanovas, The Generalized Hybrid Averaging Operator and its Application in Decision Making, Journal of Quantitative Methods for Economics and Business Administration 9(2010) 69-84.

22. J.M. Merigó, M. Casanovas, Fuzzy generalized hybrid aggregation operators and its application in decision making, International Journal of Fuzzy Systems 12(1) (2010) 15-24.

23. R.R. Yager, The power average operator, IEEE Transactions on Systems, Man, and Cybernetics - Part A: Systems and Humans 31(2001) 724-731.

24. Z.S. Xu, R.R. Yager, Power-geometric operators and their use in group decision making, IEEE Transactions on Fuzzy Systems 18(2010) 94-105

25. Z.S. Xu, Approaches to multiple attribute group decision making based on intuitionistic fuzzy power aggregation operators, Knowledge-Based Systems 24(2011) 749-760.
26. Y.J. Xu, H.M Wang, Approaches based on 2-tuple linguistic power aggregation operators for multiple attribute group decision making under linguistic environment, Applied Soft Co mputing 11(2011) 39883997. 